\title{
Bus Stops and Violence, Are Risky Places Really Risky?
}

\author{
Manne Gerell ${ }^{1}$ (D)
}

Published online: 10 April 2018

(C) The Author(s) 2018

\begin{abstract}
Geographic forecasting of crime can be done by considering prior crime or by considering spatial risk factors, e.g., using risk terrain modeling (RTM). The present paper tests both methods, but primarily focuses on RTM and on increasing our understanding of forecasting by attempting to compare the spatial risk factors for where the number of crimes is high with the spatial risk factors for where the risk of victimization is high. This is performed by fitting negative binomial models on crime around bus stops and comparing them to the same models with the number of bus passengers as exposure variable. The models also take the surrounding environment into account by fitting multi-level models with neighborhood level predictors of concentrated disadvantage and collective efficacy. The results show that some types of facilities are risk factors for crime, but not for victimization. This results in new insights into how flows of people impact on forecasting, as for instance a school is a spatial risk factor for crime, while not being associated with an elevated risk per person. The results also show that the neighborhood level of collective efficacy is a stable and significant risk factor both for crime and for risk of victimization, highlighting a potential for better crime forecasting by combining different spatial and theoretical perspectives.
\end{abstract}

Keywords RTM · Risk terrain modeling - Violent crime - Crime forecasting · Public transport . Collective efficacy

While it has been shown that proactive police patrols targeting hot spots of crime can be efficient in reducing crime (Braga et al. 2014), most hot spot policing efforts forecast crime based on prior crime, but recent research has shown that the forecasting can be improved upon by also analyzing the spatial (and temporal, e.g., Ratcliffe 2006) risk factors for crime (Drawve et al. 2016; Drawve 2016; Dugato 2013; Kennedy et al. 2011; Caplan et al. 2011). ${ }^{1}$ One method to perform such spatial analysis is risk terrain modeling (RTM), which departs from a

\footnotetext{
${ }^{1}$ Drawve (2016) for instance notes that kernel density estimation based on prior crime has the highest accuracy, but RTM has a higher reliability.
}

Manne Gerell

manne.gerell@mah.se

1 Department of Criminology, Malmö University, 20506 Malmö, Sweden 
concept of overlaying risk factors to establish a spatial risk score which can be shown on a map to indicate where crime is most likely to take place (Caplan et al. 2011). This builds on earlier work on visualizing crime risk (e.g., Bowers et al. 2004; Johnson et al. 2013), and the analysis can also be performed by methods other than RTM, ${ }^{2}$ but since this paper is written for a special issue on forecasting and RTM the choice of method was natural. RTM studies typically depart from spatial risk factors that are associated with crime based on theoretical expectations, such as retail business (Caplan et al. 2011), bars (Kennedy et al. 2011) or schools (Kennedy et al. 2016). Many of these spatial risk factors can, however, be hypothesized to impact on crime in different ways, for instance through attracting potential victims or through attracting potential offenders. To further improve crime forecasting and the preventive efforts undertaken based on forecasting, the mechanisms through which facilities are associated with crime should be better understood.

As noted by Ceccato et al. (2013) the subway stations with the highest crime counts did not have the highest crime rate per passenger. That issue is examined further in the present paper where public environment crime around local bus stops is analyzed. Spatial risk factors for crime are considered to establish whether a bus stop has an elevated risk for crime, and what the spatial risk factors for that is, but the analysis also considers whether the places have an elevated risk for victimization ${ }^{3}$ by setting the crime in relation to the number of people who board a bus at the local bus stop as a population at risk measurement. A spatial risk factor that is associated with more crime, but not a higher risk for victimization after the population at risk has been taken into account, likely functions through attracting people to the location, and is thus considered to be a crime generator (Brantingham \& Brantingham 1995). ${ }^{4}$ More people mean more potential victims and more potential offenders (Cohen \& Felson 1979; Brantingham \& Brantingham 1995). A spatial risk factor that is associated with a higher risk for victimization, however, is likely to be a risk factor with an effect over and above the effect of more people being present in the vicinity, and can thus be described as a crime attractor. Establishing the general mechanism through which a spatial risk factor may have an effect can help improve upon crime forecasting, and, importantly, it can help improve the preventive measures taken to act on forecasting to produce meaningful reductions in harm in our societies (Kennedy et al. 2016).

The present paper thus aims to add to the usability of what Kennedy et al. (2016) discuss as a vulnerability-exposure approach to crime analysis by adding a better understanding of how some risk factors contribute to crime. While Kennedy et al. (2016) examined exposure in terms of offenders by modeling the impact of prior crime using near repeats, this paper has no direct measure of such exposure, but rather tests the impact from presence of people which is a more general measure tapping into both the presence of potential offenders and of potential victims. The present paper also adds to the literature by adding to the emerging findings on importance of both micro-place features and the characteristics of the surrounding environment for RTM (Piza et al. 2016) by considering neighborhood level collective efficacy in the models. Such a

\footnotetext{
${ }^{2}$ Or under different names, a concept mentioned in Bowers et al. (2004) that is very similar to risk terrain is risk surface.

${ }^{3}$ The present study employs no individual level data, and can therefore not distinguish between a location where one individual is repeatedly victimized and a location where many individuals are victimized once each. This means that with the terms used by Tricket et al. (1992) the present study is actually dealing with crime incidence, rather than crime prevalence.

${ }^{4}$ Or, by simply being placed in a location which already attracted lots of people. Selection effects are somewhat understudied in spatial criminology.
} 
combination of theoretical constructs is in line with the the findings of Stucky and Ottensman (2009) who noted that disadvantage as a proxy for social disorganization conditioned the association of land use with crime. This is also in line with future directions for research on public transport and crime suggested by Newton (2014). Additional aims of the paper include getting a better understanding for violent crime related to public transport, and more generally for where the actual risk of violent victimization is greatest in the city of Malmö, Sweden.

The aim of this paper is thus to add to the crime forecasting literature by considering differences between risk factors for crime and risk factors for crime per population at risk. The paper uses the methodological concept of risk terrain modeling (RTM), but for forecasting purposes the findings should be relevant more generally.

\section{Risk Terrain Modeling}

RTM is a fairly straight forward method to overlay risk factors to create, and visualize, a composite risk score which can tell us whether a particular place has a high risk of crime or not. While the use of a combination of spatial risk factors to establish a general risk has been employed in numerous studies, the RTM approach has a stronger focus on visualization and usability outside of the academic world which yields a better possibility for practical adaption among police agencies or other authorities (Caplan et al. 2014a). RTM employs raster grids, dividing a city into small cells which are then assigned a risk score based on the presence (or proximity) of known spatial risk factors, such as bars, schools or ATMs (Caplan et al. 2011). The risk factors are often determined and/or weighted based on a regression or some other technique to ascertain how important they are for the crime outcome to be studied, and the resulting map of a city thus shows the relative risk for crime in each of the cells the city has been divided into. RTM has been tested on different crimes, including gun violence (Caplan et al. 2011; Kennedy et al. 2011), gun crime (Drawve et al. 2016), assault (Kennedy et al. 2016), burglary (Moreto et al. 2013), violence against the police (Caplan et al. 2014b), and motor vehicle theft (Piza et al. 2016).

RTM studies have also started to look more closely at how spatial risk factors interact to generate crime. Piza et al. (2016) for instance noted that while there are city wide risk factors there is also significant variation between different types of neighborhoods in their impact. For instance it was noted that the association of a park with motor vehicle theft was seven times greater in neighborhoods with a high share of young males in the population (Piza et al. 2016). This highlights the importance of taking account both of the spatial micro-place influences and the surrounding environment to gain a good understanding of spatial risk for crime. RTM has also been analyzed in relation to near repeat patterns to establish where near repeats are more likely to occur (Moreto et al. 2013; Kennedy et al. 2016). Near repeat patterns are crimes that follow closely on an initial incident in both time and space, and which can be modeled similarly to how deceases spread. It was noted that the RTM risk score was associated with a higher risk for a near repeat after control for the presence of the initial event; thus, suggesting that a higher risk score is associated both with a higher risk of a crime, and a higher risk for a follow up crime incident (Moreto et al. 2013; Kennedy et al. 2016). Using dynamic risk scores based on near repeats is a key venue for improvement of crime forecasting. Similar findings have also been established with a method called prospective mapping, which includes near repeat patterns combined with the presence of buildings and roads (Johnson et al. 2009), and crime forecasting has also been tested using near repeats alone (Bowers et al. 2004). 
The present study can be considered in light of such theoretical-methodological advancements of risk terrain modeling in that it considers risk terrain from a different perspective, looking at the risk for victimization in addition to the risk for crime, in the setting of public transportation and the volumes of passengers present at each location. In addition it builds on the findings of Piza et al. (2016) and Ceccato et al. (2013) that suggested potential benefits from including the neighborhood level variable of collective efficacy, by employing mixed effects models of bus stops nested in neighborhoods with concentrated disadvantage and collective efficacy as neighborhood level variables. As the bus stop data was only available for a full year it was however not possible to include near repeat patterning or similarly dynamic models into the present study.

\section{Public Transport Crime and Fear of Crime}

Public transport is an important common good, and the issue of (perceived) security on public transport has been described as one of the main factors determining its use (Newton 2008; Newton 2014). Understanding crime associated with public transport is therefore an important issue, and while it is a topic that has received a fair bit of attention both in Sweden (Ceccato \& Uittenbogaard 2014; Ceccato et al. 2013) and internationally (Newton 2008; Newton \& Bowers 2007; Loukaitou-Sideris 1999; Block \& Davis 1996; Felson et al. 1996) much remains to be learnt. More specifically, it has been argued that bus transportation in particular has received little attention during the 2000s (Hart \& Miete 2014). In the present paper the topic of public transport and crime is approached with an aim to increase our ability to forecast crime by analyzing differences in geographic risk factors when the number of crimes around a bus stop is compared to the number of crimes per passenger at a bus stop.

It is well established that important public transport nodes, or more generally places with many bus stops, tend to have high levels of crime (Brantingham \& Brantingham 1995; Weisburd et al. 2014; Ceccato \& Uittenbogaard 2014; Bernasco \& Block 2011), but as noted by Ceccato et al. (2013) this appear to largely be an effect of the number of people, at least for violence. In their study they showed that violent crime per passenger actually was higher the further away from the city center a station was positioned, while the opposite held true for theft (Ceccato et al. 2013). Subway stations with high rates of violence were additionally characterized by having ATMs, having dark corners, having high visibility in lounges, and by having more platforms (Ceccato et al. 2013). On the topic of crime related to busses, Hart and Miete (2014) found that robberies tend to cluster around combinations of spatial risk factors, with the presence of a bus stop being one of the more important risk factors. They, however, also noted some variability in the importance of bus stops for different spatial risk profiles, pointing toward interaction effects where specific combinations of risk factors may be more important than the risk factors in themselves. In some configurations of spatial risk factors a bus stop was associated with a large increase in the expected number of robberies, but in other configurations a bus stop was associated with a large decrease in the expected number of robberies.

Further, it has been shown that low-crime bus stops, as compared to high crime bus stops, were noted for better surveillance opportunities and fewer environmentally negative attributes (Loukaitou-Sideris 1999). A paper on bus route crime noted that there were more crimes per passenger in neighborhoods with more buildings, in neighborhoods with more deprivation and in neighborhoods with a high population turnover of Merseyside (Newton 2008). Similarly, a paper on vandalism of bus shelters noted positive associations both for neighborhood level 
variables, such as SES and share of youth, and for spatial risk factors, such as the proximity of play grounds or schools with high truancy rates (Newton \& Bowers, 2007). This suggests that both the land use and socioeconomic status of the area surrounding a bus route is of importance, and is in line with research on violence in the city of Malmö which made similar conclusions on neighborhood violent crime rates (Gerell \& Kronkvist 2017).

The issue of relative risk at bus stops can also be considered in relation to the related but conceptually different question of fear of crime. In the city of Malmö it has been shown that $10 \%$ of the population have abstained from using public transport due to fear of crime (Ivert et al. 2013), and in a case study of four neighborhoods it was noted that $10.7 \%$ of respondents considered their local bus stop to be a place to avoid due to concerns of fear (Gerell 2013). Levels of crime and disorder in general have been shown to have an impact on fear of crime, with individual victimization predictive of fear, and in particular if combined with a disordered neighborhood level context (Brunton-Smith \& Sturgis 2011). Neighborhood level studies have similarly shown that fear of crime is strongly associated with social disorganization, and as Ceccato and Uittenbogaard (2014) suggested opportunity based theories on the micro-scale can perhaps be combined with neighborhood level theories departing from a social disorganization perspective (Shaw \& MckKay 1942; Sampson \& Groves 1989; Sampson et al. 1997).

\section{Crime Pattern Theory and Spatial Risk Factors}

Considering differences between places where many crimes occur and places where many crimes per person at risk occur can theoretically be related to crime pattern theory (Brantingham \& Brantingham 1995; Brantingham \& Brantingham 1993). Crime pattern theory suggests that crime will tend to occur at activity nodes, paths between the nodes and edges between different types of areas, for instance where an industrial area meets a residential area. While both nodes and paths are useful concepts to apply to bus-related crime through bus stops and bus lines, the theory also brings up a distinction between places that are crime generators, places that attract many people and therefore also many potential offenders and victims (Cohen \& Felson 1979), and crime attractors, places that generate crime over and above the effect of the number of people present at the location (Brantingham \& Brantingham 1995). A crime attractor creates opportunities for crime which draws more motivated offenders to the location, and although some types of locations may act as both a crime generator and a crime attractors (e.g., a shopping mall, Brantingham \& Brantingham 1995) the distinction is useful to understand why a location has a lot of crime, and in particular how it can be prevented.

In relation to the present study, a geographic risk factor that is associated with high counts of crime, but not with high counts of crime per passenger could be considered a crime generator, while a risk factor that is associated both with higher counts and with higher rates could be considered a crime attractor. Establishing which risk factors function largely through attracting large crowds of people and which risk factors act independently of the number of people attracted may help improve forecasting techniques, and could also inform preventive efforts.

The causal mechanisms of spatial risk factors is typically not clearly established, but departing from the routine activity theory (Cohen \& Felson 1979) they can be hypothesized to be some combination of motivated offenders, suitable victims/targets and lacking guardianship. This however also implies some interaction effects, where for instance a place with lots of people such as the city train station will have lots of 
potential victims, lots of potential offenders, but also lots of guardians that possibly can be capable of preventing crime from happening.

Some types of spatial risk factors are often considered in terms of lacking guardianship, for instance foreclosures have been hypothesized to yield more battery against police due to a lack of guardianship (Caplan et al. 2014b). Guardianship can also more broadly be associated with neighborhood level collective efficacy, the combination of trust and shared expectations for informal social control within a neighborhood (Sampson et al. 1997). In order to test such a proposition the present paper employs multilevel models of micro-places nested in neighborhoods to test whether the potential guardianship effect of collective efficacy may have an impact on the spatial risk scores of micro-places. This is a research design that is similar to that of Stucky and Ottensman (2009) who combine data of land use with data on socioeconomic status as a proxy for social disorganization, but in the present study nested models are used rather than assigning all micro-places the value of its neighborhood(s).

The risk factors employed in the present study resemble the risk factors used in prior studies employing RTM (See Piza et al. 2016 for an overview). Schools, ATMs, bars, and bus stops were all included in a RTM for aggravated assaults in Chicago (Kennedy et al. 2016), and the present study additionally looks at restaurants and high schools as separate categories combined with data from a systematic social observation (SSO) of bus stops to yield data over visually identifiable markers of the terrain and its land use.

\section{Research Design}

The present paper includes two complementary analyses. The first part considers how geographic risk factors vary when trying to establish where the count of crime is high as compared to where the risk for victimization is high. In this analysis only areas around bus stops are considered, and the risk for victimization is modeled by using the number of bus passengers at a bus stop as exposure variable. In the second part a very basic RTM is performed based on the findings in the first part to test whether taking the number of bus passengers into account instead of traditional risk factors in RTM is a viable way to understand where crime is likely to take place. In addition a number of sensitivity tests are considered in order to ascertain the stability of the results.

The first part deviates from traditional RTM analysis in only considering parts of the city near a bus stop. This is similar to, but a much more drastic reduction of locations than, how Moreto et al. (2013) removed all pixels not containing a street segment in order to reduce the bias introduced of cells with no street since crimes are coded to street addresses. The outcome of public environment violence is studied in an attempt to find out both where the risky places in the city are, and where places with a high risk of public violent victimization in the city are located, in addition to understanding the correlates of those types of places. The outcome variable of public environment violent crime uses data over robbery and (aggravated) assault that the police have coded as taking place in public environments. The data was spatially matched by using buffers around the bus stops of 50 and $200 \mathrm{~m}$, and joining them to $200 \mathrm{~m}$ buffers around the independent variables of restaurants, bars, ATMs, schools, and high schools. To determine risk scores for risky places the outcome of public environment violent crime was regressed in hierarchical negative binomial models of bus stop areas nested in neighborhoods, and to determine the risk of victimization the same type of models were run with the number of bus passengers at the location as exposure variable. While the use of 
negative binomial regression for RTM has been suggested as appropriate (Piza 2012; Drawve et al. 2016; Moreto et al. 2013), most prior studies have not employed multi-level models. ${ }^{5}$ All models were also fitted without the nested structure, which has the advantage of not having to exclude cases due to missing neighborhood level data. The multilevel models include 487 bus stop locations while the non-multilevel models include 586 bus stop locations. To test the robustness of results models were also fitted on different buffer sizes (50 and $200 \mathrm{~m}$ ) and on assaults and robberies separately.

The second step of the analysis performs two RTMs based on the findings from the first step. One RTM uses risk factors identified to be associated with higher crime counts around bus stops, and one RTM uses risk factors associated with a higher risk for victimization around bus stops. In practice this means that the latter uses fewer geographic variables, but instead uses the number of bus passengers as a risk factor. The RTMs are fairly crude, based on assigning $50 \times 50$ meter pixels values from kernel density estimations for the independent variables and assigning all pixels in a neighborhood the value of the neighborhood variable. The specifics of the RTM is outlined in more detail in the results section below.

\section{Data}

To understand why places are risky, administrative data over restaurants, bars, nightclubs, schools, high schools, and ATMs are used. Data was linked to the bus stops by employing buffers of $200 \mathrm{~m}$ (mirroring the $600 \mathrm{ft}$ used in Drawve et al. 2016) around both the risk factors and the bus stop, and where buffers were overlapping the risk was added to the location. In addition a systematic social observation ( $\mathrm{SSO}$ ) of the bus stops was performed to gather data on the type of bus stop and the surrounding area of the bus stop. On the neighborhood level a measurement of collective efficacy was included as prior studies of public environment violence in the city of Malmö have highlighted collective efficacy as a key variable (Gerell $\&$ Kronkvist 2017). These variables resemble, but are somewhat simpler than, the variables used by Ceccato et al. (2013; Also Ceccato \& Uittenbogaard 2014) when they studied the Stockholm subway. An advantage of the present study is the availability of a direct measurement of neighborhood level collective efficacy, which is discussed by, but not included in their work (Ceccato et al. 2013).

The datasets generated during and/or analyzed during the current study are available from the corresponding author on reasonable request.

\section{Public Environment Violence}

Data from the police over public environment violence for the one year period of March 1st 2014 to February 31 st 2015 was retrieved. The data includes assaults and robberies against a person, and the main analysis was run with the combination of the two, while also testing all models with robberies and assaults separately. The assault variable includes normal assaults, someone hitting someone else, and serious assault, where a weapon is used or serious damage

\footnotetext{
5 Prior RTM studies using larger area data in combination with micro-level data have sometimes employed other methods, for instance Drawve et al. (2016) used smoothing along boundaries of block groups and assigned each cell a value. Arguably, however, a nested structure will yield a better picture by taking account of potential unmeasured neighborhood level variables that may impact on findings.
} 
inflicted. In the present study only the coordinate and type of crime was used, although data over time and date was available as well. As shown in Fig. 1 the public environment violence is highly clustered to central parts of the city.

\section{Register Data}

Data was drawn from the municipality over the number of permits to serve alcohol valid at the 1 st of January 2014, which was divided into permits lasting to no later than 1 am to cover restaurants and permits to serve alcohol after 1 am for bars and nightclubs. School and high school locations for 2013 were also retrieved from the municipality, while ATM locations were coded based on 2017 data from the major banks of Sweden. It should be noted then that the

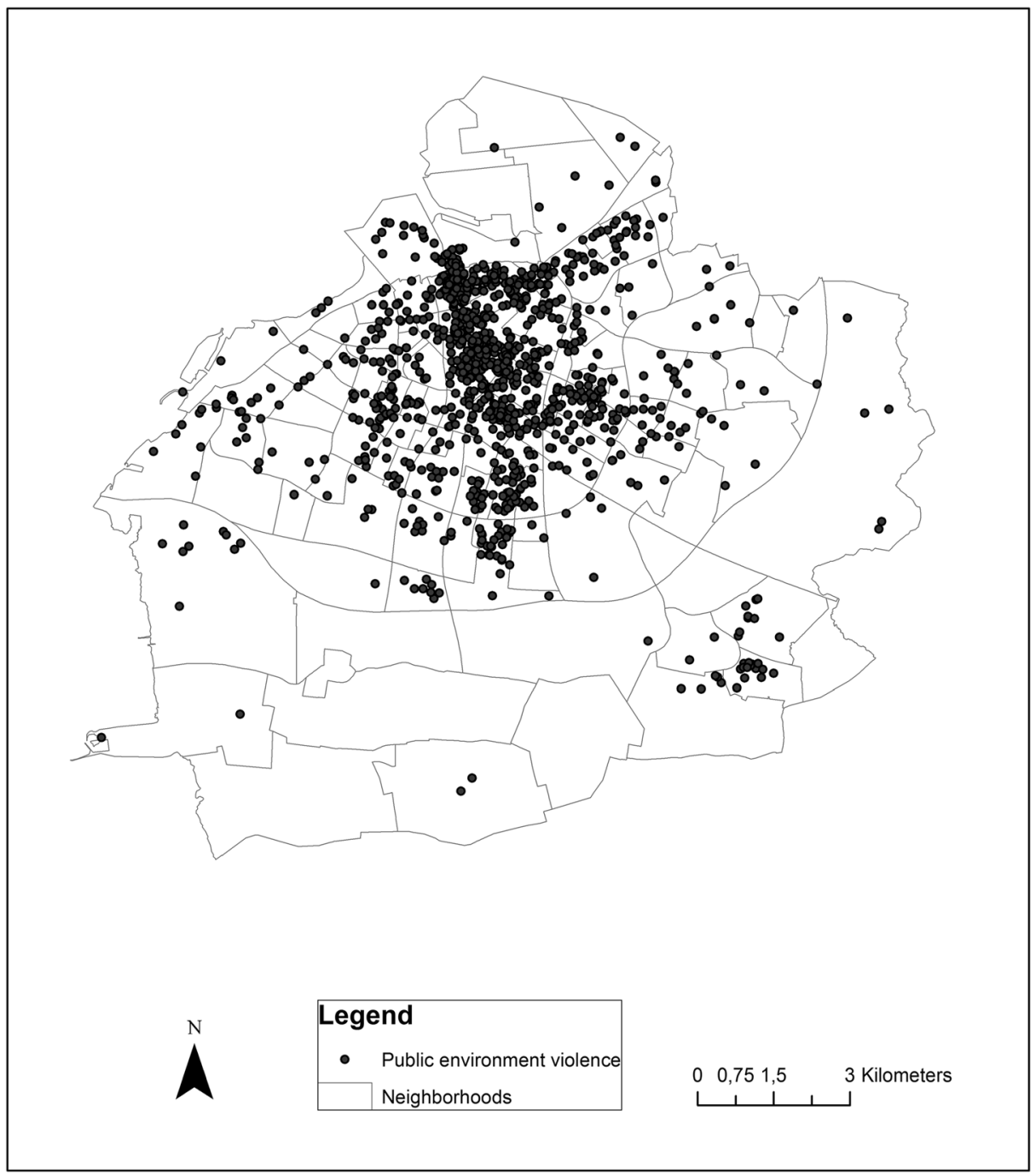

Fig. 1 Public environment violence incidents in the city of Malmö, 1st of march 2014 to 28th of February 2015 
ATM data is somewhat lacking, both due to the data retrieved being for a later year, and due to the fact that some ATMs are operated by other financial service actors than the major banks.

\section{Public Transportation Data}

Data from the county public transport company (Skånetrafiken) was retrieved over the number of started bus trips at geocoded and named bus stops during a one year time period from march 2014 to march 2015. The named bus stops in turn comprise between one and eight bus stop locations, in most cases two locations with one on each side of the street, but in some cases including several bus stops at intersections where more than one busline meet or at bus stations/squares. These more exact bus stop locations had no coordinates, and the geodata thus only includes an approximate (or average) location for the two or more actual locations where the bus stops are located. In order to increase the reliability and quality of the data a systematic social observation ( $\mathrm{SSO}$ ) of all named bus stops with at least 10,000 passengers $(N=262)$ were performed, covering a total of 586 bus stop locations (mean number of locations per named bus stop $=2.24, \min =1$, $\max =8$ ). The SSO was mostly performed using Google Street View (GSV), which for the majority of the city was available for June-July 2014 matching the data nicely. In some of the outer parts of the city, however, only data from 2011 was available at GSV, and in a few cases $2009(N=2)$. In a minority of the cases no GSV data at all was available, and in those cases $(N=26)$ the places were visited in May 2017 to gather data. It cannot be ruled out that there is some inaccuracy in the data due to the differing time periods for data collection, but the overall impact of such a bias should be small on the analysis. Since one of the aims of the present paper is to analyze bus trips as a measurement of the number of people at risk of being victimized at a location it was assumed that passengers could be divided evenly between the locations within a bus stop, e.g., it was assumed that as many people took the bus toward the city center as away from the city center. This is a generalization that warrants some caution in interpreting the results, but in many cases it can plausibly be argued that people tend to take the bus both to a location and back from it, so even if the actual number of people boarding the bus might be inaccurate, the number of people associated with the location of the bus stop would be fairly accurate. For places with more than one bus line and more than two bus stops, passengers were assigned to the exact bus stop locations based on each bus line where it was possible to determine. ${ }^{6}$ The resulting people at risk measure had a mean value per bus stop location of 56,543, with a minimum of 2126 and a maximum of $1,855,548$. During the SSO the bus lines associated with each bus stop location was also recorded, and each bus stop location was assigned a value on whether it covered one of the city's eight main bus line or one of the secondary bus lines that run less frequently and with fewer passengers.

\section{SSO Data}

While the SSO contributed with geo-data over exact bus stop locations it was also used to gather more data on the bus stops studied. Each bus stop location was coded based on the type

\footnotetext{
${ }^{6}$ Major nodes have each bus stop location assigned a letter (A, B, C, etc), and with maps showing the exact location for each of the bus stops. The passenger data had the corresponding letters identifying the locations enabling an exact number of passengers to be assigned to a bus stop location. For smaller nodes, however, this was not available, and instead the mean across bus stop locations was used.
} 
of bus stop, being a bus stop with a sign, a sign and a bench, or a shed. As the type with a sign and a bench was very rare, the models only test for an effect of a shed (as opposed to either only a sign, or a sign with a bench). In addition, the visible area surrounding the bus stop was coded into the categories of residential, commercial, other type of facilities, park/ green area, and square. Surrounding environment observations were based on looking at the bus stop and considering what could be seen surrounding it, and a bus stop location would thus be coded only based on what was on the same side of the street. These categories were not mutually exclusive, and while most locations were coded as just one type of environment many also had more than one. Residential was coded when residential buildings were dominating. Commercial was coded if at least one restaurant or store was visible. Other type of facility was coded when office buildings, industries, schools or other non-residential and non-commercial buildings were visible. Park/green area was coded if either only greenery was visible (commonly with large bushes/trees hiding whatever was behind it) or when a large green area was visible. Finally, square was coded when a large open area that was not green was clearly visible. Descriptive statistics from the SSO are reported in Appendix Table 4.

\section{Neighborhood Level Data}

As it has previously been shown that collective efficacy (e.g., Sampson et al. 1997) has a strong association with public environment violence in the city of Malmö (Gerell \& Kronkvist 2017), I include a measurement of collective efficacy from the 2012 Malmö community survey $(N=4195)$ in the models. The survey comprised 104 neighborhoods, but neighborhoods with few respondents and/or residents were excluded, and additionally some neighborhoods had no bus stop with more than 10,000 passengers registered and were thus excluded for a total sample of 89 neighborhoods used in the present study. The collective efficacy index is based on five items each for cohesion/trust and for informal social control which exhibits a high degree of internal consistency (Cronbach alpha $=0.89$ ).

To further test the associations, neighborhood level concentrated disadvantage has also been included in the study, comprising a factor-score adjusted index of reverse coded median income, share on public assistance, share unemployed, share foreign born, persons per room, and share single parents (Cronbach alpha $=.95$; see Gerell \& Kronkvist 2017 for more discussion). This index is very similar to that of Stucky and Ottensman (2009) in their study of spatial risk factors for violent crime.

\section{Results}

In the first step of the analysis, bivariate correlations between the independent variables was established both for the count of violence around a bus stop and for violence per passenger around a bus stop (Table 1). For the count of violence, all variables showed statistically significant associations with the outcome. Residential buildings or buildings that were not residential, nor commercial were associated with fewer crimes, as were parks. All other variables were associated with more crimes, in accordance with expectations. When the outcome was transformed into crimes per bus passenger, however, several variables lost significance. The remaining significant variables (all positively associated with crime per passenger) were square, restaurants, bars, ATMs, schools, and high schools. 
The main models presented use $200 \mathrm{~m}$ buffers around the bus stop to identify crimes and independent variables supposed to have an impact on crime around bus stops. Table 2 reports the results for count of crimes and Table 3 reports the results after adjusting for population at risk by setting the number of passengers as exposure variable. In the first model only registry data is used, in the second model SSO-data is included, in the 3rd model nested models are employed, and in the 4th model the neighborhood level variables of concentrated disadvantage and collective efficacy are included. Results from Table 2 shows restaurants and schools are associated with more violence in model 1. In model 2 additional variables turn out significant, bus stops with a roof and near squares have more violence, while bus stops in parks or green areas have less violence. Model 3 takes the nested structure of the data into account by considering that the outcome of violence may be related to the surrounding neighborhood and yields several differences. Restaurants and squares are no longer significant, while bars and ATMs are. In model 4 when neighborhood level variables are added restaurants return to significance, while ATMs drop below the threshold.

While the inconsistent results may appear strange it should be noted that the variables that exhibit such inconsistencies all are closely associated with each other. Restaurants, bars, ATMs, and squares are highly correlated, and in practice they can all be said to signify some sort of local center. This is discussed and explored in more detail below in the section for sensitivity tests.

In Table 3 the same models are run, but this time including the number of bus passengers at the bus stop as exposure variable. This results in most variables no longer being significant. In the first model, which is run as a single level negative binomial regression, restaurants and schools are associated with higher risk, while high schools are associated with lower risk. In model 2 the SSO data is added, with the only substantial difference being that high schools no longer are significantly associated with less crime risk. When the nested structure of the data is accounted for in a multilevel model however, only the presence of restaurants remains significant. When neighborhood level variables are added in model 4, collective efficacy is significantly associated with risk for victimization. The final model thus shows that bus stops in low collective efficacy neighborhoods and near restaurants tend to exhibit higher risks for violence.

Due to the importance of nodes for public transportation where many bus lines intersect with each other and with regional busses and/or trains, a dummy variable was inserted to test whether nodes exhibit some particular characteristics. Nodes have no impact on the count of

Table 1 Bivariate correlates of bus stop violent crime counts and bus stop violent crimes per passengers

\begin{tabular}{lcc}
\hline & Count of violence & Violence per population at risk \\
\hline SSO: bus stop with roof & $.163^{* *}$ & -.02 \\
SSO: residential & $-.120^{* *}$ & -.025 \\
SSO: commercial & $.121^{* *}$ & .078 \\
SSO: other & $-.085^{*}$ & -.015 \\
SSO: park & $-.169 * *$ & -.075 \\
SSO: square & $.589 * *$ & $.134^{* *}$ \\
Restaurants & $.721^{* *}$ & $.315^{* *}$ \\
Bars & $.607 * *$ & $.155^{* *}$ \\
ATMs & $.524 * *$ & $.134^{* *}$ \\
Schools & $.083^{*}$ & $.095^{*}$ \\
High schools & $.259 * *$ & $.105^{*}$ \\
\hline
\end{tabular}


Table 2 Negative binomial regression of violent crime around bus stops

\begin{tabular}{lllll}
\hline & $\begin{array}{l}\text { Model 1. } \\
\text { Count of } \\
\text { violence }\end{array}$ & $\begin{array}{l}\text { Model 2. Count of violence } \\
\text { including SSO variables }\end{array}$ & $\begin{array}{l}\text { Model 3. Count of } \\
\text { violence (nested } \\
\text { data) }\end{array}$ & $\begin{array}{l}\text { Model 4. Count of } \\
\text { violence (nested } \\
\text { data) with CD \& } \\
\text { CE }\end{array}$ \\
\hline Restaurants & $.071^{* * *}$ & $.061^{* * * *}$ & .019 & $.023^{*}$ \\
Bars & -.004 & -.024 & $.043^{*}$ & $.044^{*}$ \\
ATMs & .007 & .015 & $.116^{*}$ & .071 \\
Schools & $.416^{* * *}$ & $.426^{* * *}$ & $.201^{* *}$ & $.207^{* *}$ \\
High schools & -.219 & -.035 & .175 & .165 \\
SSO: bus stop with & & $.400^{* * *}$ & $.166^{*}$ & $.169^{*}$ \\
$\quad$ roof & & .080 & .056 & .017 \\
SSO: residential & & .106 & .048 & .067 \\
SSO: commercial & & -.315 & -.081 & -.123 \\
SSO: other & $-.324^{*}$ & $-.287^{* *}$ & $-.286^{* *}$ \\
SSO: park & $.581^{*}$ & .289 & .276 \\
SSO: square & & & .225 \\
Neighborhood: & & & & \\
$\quad$ concentrated & & & & $-.873^{* * *}$ \\
disadvantage & & & & \\
Neighborhood: & & & & \\
$\quad$ collective efficacy & & & & \\
\hline
\end{tabular}

violence models, but are significantly associated with lower risk for victimization (coeff -0.92 , $p=0.006$ ) while having no substantial impact on the other variables. This suggests that the nodes for public transportation, while typically having a high crime count, in fact can be described as safer than other bus stops.

Taken together these results suggest that some of the risk factors commonly spatially associated with crime largely function through drawing more people to a location. The presence of a school for instance is associated with more crime, but that association is much weaker when adjusting for population at risk, and thus it appears to largely be functioning as a crime generator through drawing people to the location as opposed to a crime attractor which

Table 3 Negative binomial regression of violent crime around bus stops with bus passengers as exposure variable

\begin{tabular}{lllll}
\hline & $\begin{array}{l}\text { Risk of } \\
\text { violence }\end{array}$ & $\begin{array}{l}\text { Risk of violence } \\
\text { including SSO } \\
\text { variables }\end{array}$ & $\begin{array}{l}\text { Risk of violence } \\
\text { (nested data) }\end{array}$ & $\begin{array}{l}\text { Risk of violence } \\
\text { (nested data) with } \\
\text { CD \& CE }\end{array}$ \\
\hline Restaurants & $.069^{* * *}$ & $.067 * * *$ & $.037 *$ & $.029^{*}$ \\
Bars & -.042 & -.042 & .000 & .014 \\
ATMs & -.121 & -.124 & -.004 & -.059 \\
Schools & $.275^{* * *}$ & $.280^{*}$ & .103 & .105 \\
High schools & $-.302^{*}$ & -.293 & .036 & .037 \\
SSO: bus stop with roof & & .040 & .022 & -.044 \\
SSO: residential & & .012 & .029 & -.016 \\
SSO: commercial & .076 & .010 & .016 \\
SSO: other & & -.088 & -.110 & .056 \\
SSO: park & -.164 & -.235 & -.205 \\
SSO: square & .005 & & -.116 \\
Neighborhood: concentrated & & & & .091 \\
$\quad$ disadvantage & & & & $-.706^{* * *}$ \\
Neighborhood: collective & & & & \\
$\quad$ efficacy & & & &
\end{tabular}


yields crime over and above the effect from more people. In terms of RTM this may point to a possibility of using a population at risk measurement directly as a risk factor for crime.

In the final step of analysis, therefore, RTM maps have been produced in ARCMap that depart from these results. The first map uses four risk factors associated with more crime around bus stops (Table 2, model 4); restaurants, bars, schools, and low collective efficacy neighborhoods. The SSO data was excluded as the map will cover the whole city and not just the bus stop locations. The second map uses the two risk factors associated with a higher risk for violent victimization (model 4, Table 3) in addition to a third risk factor made up of the number of bus passengers. For the point data (restaurants, bars, schools, and bus stops) kernel density estimations $(50 \mathrm{~m}$ pixel size, $500 \mathrm{~m}$ radius, processing extent set to municipal boundaries) were used to assign pixels a value for each risk factor. The rasters were reclassified into risk scores of 1-5 using quantiles. The collective efficacy neighborhood data (polygon data) was used to assign each pixel (based on center of pixel) the value of the neighborhood it is located in, and reclassifying it into five categories based on equal intervals (since the data was already standardized into Z-scores) after reverse coding it to yield a positive relationship with violence. The rasters were then summed into a composite raster, and divided into four risk score ranges based on equal intervals to allow for comparison. This is a very crude RTM, but serves a general purpose of mapping risk with and without taking the population at risk into account. The resulting maps are shown in Fig. $2 \mathrm{a}$ and $\mathrm{b}$ ( $2 \mathrm{a}$ on the left uses traditional RTM, $2 \mathrm{~b}$ on the right uses bus passenger data instead of two risk factors).

Due to higher maximum values in the traditional RTM map (left) neighborhoods with low collective efficacy but no other risk factors end up being coded as yellow (medium risk), while this method results in fewer locations recorded as medium-high and high risk locations. As can be noted by comparing the RTM maps with the map of violence (Fig. 1) both RTM outputs appear to be fairly good at explaining risk. To more formally compare the two RTMs the medium-high to high risk pixels were extracted, and the resulting new raster was converted to polygons which then were used to consider how many of the crimes that fall within the polygons in relation to their share of the municipal area. Out of 1923 violent crimes, 1410
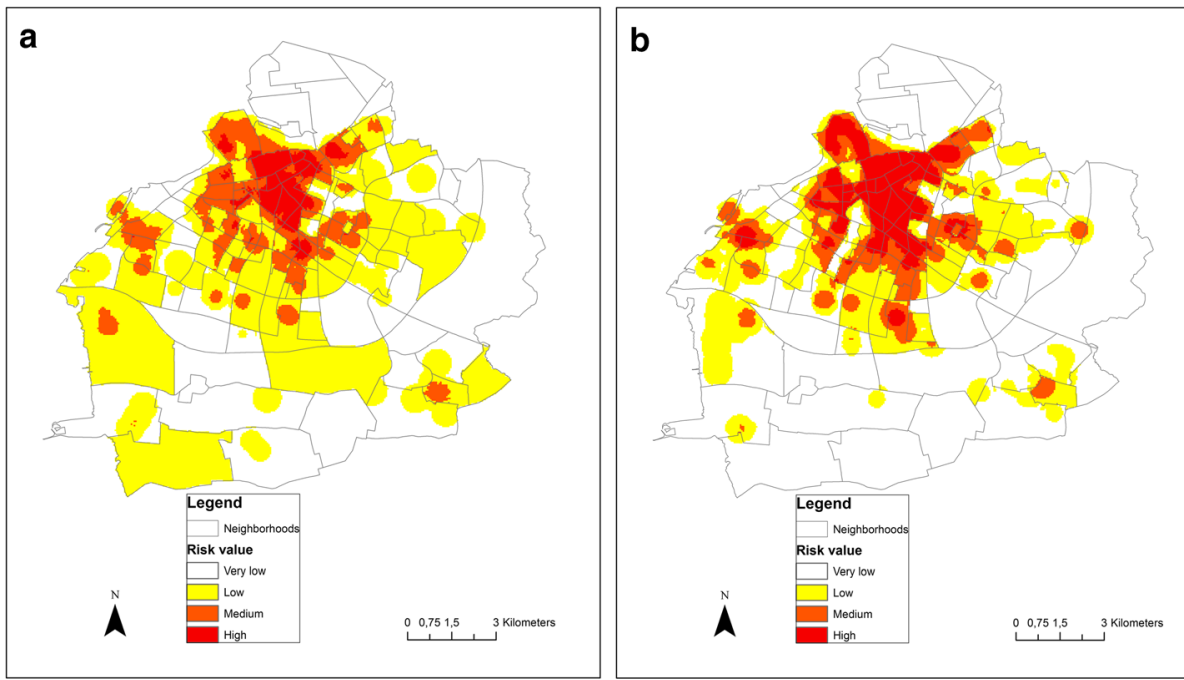

Fig. 2 a (left) RTM map using traditional geographical risk factors based on Table 2. b (right) RTM map using bus passenger data and variables based on Table 3 
(73\%) were identified with the traditional RTM while $1565(81 \%)$ were identified with the method taking bus trips into account. This is reflected by the higher area covered, and counted as crimes per area identified the traditional RTM (12\% of the city's area) method was better with 70 crimes per square kilometer of medium to high risk, while the bus passenger based method (16\% of city area) only achieved 60 crimes per square kilometer.

To further assess these results they were also compared with a kernel density estimation of prior violence to forecast crime. The kernel density was based on a $500 \mathrm{~m}$ radius, $50 \mathrm{~m}$ pixel size, estimation of public environment assaults and robberies from 2013. A quantile distribution of the resulting raster in four score ranges was then used similarly to the risk estimation for the RTM, and the two highest risk score ranges were extracted and matched with the public environment violence data used as outcome in the present study. The resulting forecast yields a very high 1799 out of 1923 incidents (93.6\%) correctly forecast, but on a much bigger area ( $21.8 \%$ of city area), and 51 crimes per square kilometer (map not shown). Of the three methods used, it can be noted that the bigger the high-risk area, the higher the share of violence correctly forecast, but with a lower rate of violence per square kilometer correctly forecasted. It is not possible to draw any firm conclusions on which of the methods is better at forecasting based on this fairly crude analysis, but rather the main take away should be that all three methods are reasonably good at forecasting future public environment violence. The density estimation of prior violence identified the most incidents, but the lowest rate of incidents per area. Increasing the threshold for areas that are identified as high risk would be likely to reduce the number of forecast incidents, but also to increase the rate of correctly forecast incidents per square kilometer. For policing purposes a higher rate of correctly identified incidents per area may well be of higher importance to enable effective policing, but identifying optimal cut off points is beyond the scope of this paper. The basic forecasting estimation tests employed here used fairly arbitrary cut off points and no weighting of the variables, but serve to illustrate the viability of different methods to forecast crime. The focus of this paper is on the use of population at risk data to improve on forecasting and its application in crime prevention. The results do not show that population at risk data appear to yield a better forecasting hit rate, but it may give a better understanding of why some locations have high levels of violence, and thus better possibilities of preventing it. Some facilities yield more crime simply because they are associated with more people, while other facilities yield more crime over and above the effect of more people being present.

\section{Sensitivity Tests}

To test the robustness of the results the regression models were fitted using $50 \mathrm{~m}$ buffers around bus stops instead of $200 \mathrm{~m}$ as well. This results in much less variation in both the dependent and independent variables (e.g., lower reliability of results), but on the other hand gives a higher validity as the most immediate surroundings of a bus stop should be more closely related to the characteristics of the bus stop and the risk faced by bus passengers there. The results are similar in the first few steps of modeling (results not shown), with restaurants and schools showing significant associations with violence (in addition to collective efficacy in the mixed models), but once population at risk is accounted for only collective efficacy remains significant. This again points to the stability of restaurants and schools as predictors of where crime occurs, and underscores the strong association of collective efficacy with both crime and risk for victimization. 
The models were also run on assault and robbery separately, which yields some differing results while not changing the main conclusions of the paper. For counts of robberies schools loose significance, while the land use variable capturing non-residential and non-commercial facilities is noted for a significant negative association in the final model. For counts of assault, bars and bus stop with roof loose significance while high schools and neighborhoods with higher levels of disadvantage are noted for positive associations with violence. When taking population at risk into account the results are substantially unchanged for both robberies and assaults as compared to the combined measure reported in Table 3.

Since restaurant was a stable predictor, but also highly correlated with several other variables that theoretically are expected to impact on crime, the models were also run without the restaurant variable. In the models of crime count this has a fairly big impact, pushing square, ATM, and high school to significant values, suggesting that the multicollinearity issues of the main model may be hiding some important associations. When adjusting the models for population at risk, however, this has no substantial impact, although it does increase the coefficients for bar, ATM, high school, and square a bit none of them reach significance (Appendix Table 5). To further explore potential issues with multi-colinearity multiple other models were also tested. Excluding squares, ATMs or bars from the count models have no substantial impact on the results. While the variables are associated with each other and all tend to signify a local center, restaurants are by far the most stable of these predictors for violence in the city of Malmö.

Finally, since some bus stops located in very central locations were only serviced by secondary bus lines the analysis was also run using main bus lines only (lines 1 through 8 , with secondary bus lines having two digit identifiers). For the final models of Tables 2 and 3 respectively this yields some notable differences. Regressed on the count of violence this results in restaurants and schools no longer being significant, though for schools it is close $(p=$ $0.05)$. Similarly, for the population-adjusted regressions in Table 3 the mainline-only fitting of the final model results in collective efficacy being the only significant variable. While not significant $(p=0.081)$ this model also yields a much higher coefficient for bars on the expense of restaurants. The association of restaurants with public environment violence thus appears to be driven by the bus lines that go to less populated areas.

\section{Discussion and Conclusion}

The main finding of this paper is that many of the risk factors for violent crime appear not to be risk factors for violent victimization, which has implications for our use of crime forecasting based on spatial risk factors in crime prevention. A node for public transportation for instance has high levels of violence, but low risks of victimization when violence is considered in relation to the number of bus passengers at the location. Regarding where violence is concentrated, it is noted that high levels of public environment violent crime tend to cluster around facilities that signify (local) centers, with modeling to a varying degree finding that some or all of the variables restaurant, bar/nightclub, and ATM are significantly associated with violence in addition to the presence of a square. Out of these variables the restaurant variable has the most stable association with violent crime, but as noted in the sensitivity analysis this seems to be driven by violence around bus stops at secondary bus lines that frequent less populated areas of the city. 
When taking the surrounding neighborhood into account the variable with the most stable and substantial association with violence turns out to be neighborhood level collective efficacy (e.g., Sampson et al. 1997). There is much more violence around bus stops that are located in neighborhoods with low collective efficacy, that is, where the residents express a lower trust in their neighbors and lower expectations that their neighbors will intervene against crime or unwanted behavior. Most of the models on violence count in addition note that there is less violence around bus stops located around parks. While this in part signifies the fact that such bus stops tend to have fewer passengers, and thus fewer potential victims and offenders, it is still a somewhat interesting finding. Parks could be expected to yield a lower degree of natural surveillance as there are fewer people around, and could therefore be expected to be more dangerous places. When taking the population at risk into account parks, however, are registered for non-significant negative associations with violence. While this seems to suggest that park areas certainly are not more dangerous than other places, some caution is advised in interpreting these results as parks also will tend to suffer from a lower spatial reliability of police data due to the lack of addresses where crimes can be recorded.

The findings of this paper on geographic risk factors for violence largely corroborate prior findings regarding forecasting of where violence occurs, but it also shows that much of the association from some risk factors appear to be related to the number of people around the location rather than to some intrinsic value of the facility measured. There is more violence near schools, but that appears to be driven by the fact that there are more people around schools rather than the schools producing more violence per person visiting the area. This suggests that some risk factors act more as crime generators, generating opportunities for crime by drawing victims and offenders alike to the location, rather than crime attractors, which attract offenders to the location for the purpose of committing a crime, prior research has shown that this may vary by time of day (Smith \& Cornish 2006 cited in Newton 2014). Further research is needed to uncover the extent to which spatial risk factors act as crime attractors, crime generators, or both. The RTM performed in the present study further seem to suggest that incorporating bus passenger flows into the RTM does not increase its accuracy, and that forecasting based on prior violence similarly yields as good a hit rate, but it should be noted that the RTM employed in the present study was very crude, and further elaboration on the issue is needed to make any definitive statements on the topic based on this data. All three methods tested to forecast crime in the present paper produced reasonable hit rates, but as this paper also has shown an analysis that takes population at risk into account can help shed some more light on why some facilities are associated with violence.

It should also be noted that bus stops are allocated based on where perceived needs for such services may arise. While Ceccato et al. (2013) note that subway stations in Stockholm were planned first, and neighborhoods followed, the process may be more dynamic for bus stops which represent a much smaller investment than a subway. Causal effects can be hypothesized to exist in both directions between bus stop locations and other services. A bus stop will be more likely to be allocated to where there already are restaurants, but restaurants will likely also be more likely to be allocated to places with good public transport. Selection effects may thus be at play here, an issue which warrants further attention from the field of geographic criminology. Where a restaurant (bus stop, bar, school) is allocated is not random, and whether the association of a restaurant with crime is causal or dependent on underlying variables is rarely addressed. While the present study indirectly addresses this from one perspective, through the control for population at risk, it cannot say anything about causality. More experimental evidence is needed to move the issue further, one example of which that like 
this study incorporates the concept of collective efficacy is the natural experiment of Cerda and colleagues (2012). They find that transit oriented development in Medellin, Colombia, was associated with decreased violence (Cerda et al. 2012). In cases where experimental studies are not viable, the present study supports the findings of Ceccato et al. (2013) who argue that passenger flow data can inform our understanding of why some locations have more crime, and that high volume locations tend to be associated with lower crime risks, possibly due to better guardianship associated with the steady flow of people.

The sensitivity test showing that the association of restaurants with violence is driven by the secondary, less frequent, bus lines can possibly be interpreted in terms of small, local, centers which usually have a restaurant, and possibly attract more violent people. The risk for violent victimization is thus highest in local centers of disadvantaged neighborhoods having low collective efficacy. Additionally, the risk for violent victimization is significantly lower in major nodes of public transport, confirming the findings of Ceccato et al. (2013) that made a similar finding by considering distance to the city center. This is of interest in relation to public transport as a common good, as the nodes where large flows of people pass through appear to be relatively secure.

Disadvantage has no effect on the combined measure of violence once collective efficacy is taken into account, but it is associated with higher counts of assaults. Disadvantage is also strongly associated with collective efficacy and as availability of collective efficacy data may vary across cities disadvantage can be used as a proxy for identifying such locations in crime forecasting. ${ }^{7}$ This suggests that both the surrounding neighborhood and the specific location need to be considered to understand crime as suggested by Newton (2014) in his summary of public transport and crime research. While this paper uses a multi-level design to take account of both neighborhood (meso) and place-specific (micro) level variables, the analysis did not incorporate interaction effects. Interaction effects are likely to be present, as suggested by Hart \& Mieke (2014) who noted that crime around bus stops occurs when specific combinations of risk factors are present rather than risk of crime increasing statically with the presence of a specific risk factor. While not studying public transport, such interaction effects were indeed identified by Stucky and Ottensman (2009), with the impact of land use variables conditioned on disadvantage and other variables that can be related to social disorganization theory. The association of commercial or industrial lands, for instance, depended on the level of disadvantage in the neighborhood, with more disadvantage resulting in stronger effects from the land use variables. Theoretically it appears plausible that similar associations are at place around the bus stops in Malmö too, with socially disorganized neighborhoods impacted more by the presence of a restaurant or bar that may draw rowdy people to its vicinity. More generally such interactions have previously been suggested to understand crime in Chicago (St Jean 2008). This may also be related to the connectivity of bus stops in the bus network, with bus stops that are important nodes in the bus network potentially having a different association with land use risk factors than more peripheral bus stops. Employing network analysis on the bus stop network could shed more light on such interactions in future research.

Future research in addition needs to further consider dynamic risk factors. As noted in the introduction some work on the combination of near repeat patterns and RTM has emerged (Bernasco et al. 2009; Moreto et al. 2013; Kennedy et al. 2016), and combining the two is

\footnotetext{
${ }^{7}$ While those results are not presented in the paper, concentrated disadvantage tend to be highly significant if collective efficacy is not included in the models.
} 
likely to result in much better forecasting. Some places have a higher risk for crime, but that risk is also time-variant. A recent example of this is how scaffolding appears to have contributed to a large, but temporary, spike in burglaries in a neighborhood undergoing renovation in the city of Malmö. The spike was large enough to result in a city-wide increase in near repeat patterning (Hoppe \& Gerell 2018). Similar to the above discussion on disadvantage and restaurants, there may be interaction effects at work, as the particular neighborhood at hand also can be considered socially disorganized. Would scaffolding have had the same effect in a more affluent neighborhood? The combination of spatial risk factors with a dynamic view on risk associated with prior crime holds some promise to investigate such issues and yield better opportunities for crime prevention. As noted by Johnson et al. (2013) prospective mapping could be used to identify high risk areas and target those with preventive efforts. This has also recently been tested in a randomized controlled trial that found burglaries could, to some extent, be prevented with a target hardening intervention (Johnson et al. 2017). The question raised by Bowers et al. (2004), on whether dynamic risk models was the future of crime mapping should be answered affirmatively, and it is perhaps surprising that not more has happened on this topic since their paper was written.

The findings of this paper have some implications for forecasting and RTM, and in particular for preventive efforts based on such analysis. It also raises an interesting question on how to prioritize; crime prevention is most effective at locations with high volumes of crime (Abt 2017), but there could be an argument made to additionally focus efforts at locations where the risk of victimization is high. Preventive efforts directed at high risk for victimization locations, however, will likely produce lower effects due to the lower volumes of crime, unless much more directed preventive efforts can be used to counteract such a lowering of the impact. In order to facilitate more precise preventive efforts it will be important to understand the mechanisms through which spatial risk factors interact to generate opportunities for crime, and this paper is but a tiny piece of such a puzzle where much work remains both in terms of outlining the mechanisms to improve forecasting and in terms of developing crime prevention to actively work on dealing with such mechanisms to reduce crime.

Acknowledgements This research was done in collaboration with K2 - The Swedish Knowledge Centre for Public Transport. The author would like to thank Andrew Newton from the University of Huddersfield for helpful suggestions and advice.

\section{Appendix}

Table 4 Descriptive statistics from the SSO

\begin{tabular}{lr}
\hline & Share \\
\hline Bus stop with sign & $41.3 \%$ \\
Sign and bench & $0.9 \%$ \\
Roof & $57.8 \%$ \\
Residential surroundings & $49.7 \%$ \\
Commercial & $16.2 \%$ \\
Other type of facilities & $20.1 \%$ \\
Park/green area & $32.4 \%$ \\
Square & $6.1 \%$ \\
\hline
\end{tabular}


Table 5 Excluding restaurant to test sig of other variables. Final model for two outcomes

\begin{tabular}{lll}
\hline & Count of violence & Violence per population at risk \\
\hline Restaurants & - & - \\
Bars & $.062^{* * *}$ & .039 \\
ATMs & $.131^{* *}$ & .013 \\
Schools & $.209^{* *}$ & .113 \\
High schools & $.264^{* *}$ & .152 \\
SSO: Bus stop with roof & .144 & -.072 \\
SSO: residential & .009 & -.042 \\
SSO: commercial & .086 & .037 \\
SSO: other & -.124 & .043 \\
SSO: park & $-.293^{* *}$ & -.235 \\
SSO: square & $.368^{*}$ & -.015 \\
Neighborhood: concentrated disadvantage & .181 & .022 \\
Neighborhood: collective efficacy & $-.890^{* * *}$ & $-.781^{* * *}$ \\
SSO: bus stop with roof & .144 & -.072 \\
SSO: residential & .009 & -.042 \\
SSO: commercial & .086 & .037 \\
SSO: other & -.124 & .043 \\
SSO: park & $-.293^{* *}$ & -.235 \\
SSO: square & $.368^{*}$ & -.015 \\
\hline
\end{tabular}

Open Access This article is distributed under the terms of the Creative Commons Attribution 4.0 International License (http://creativecommons.org/licenses/by/4.0/), which permits unrestricted use, distribution, and reproduction in any medium, provided you give appropriate credit to the original author(s) and the source, provide a link to the Creative Commons license, and indicate if changes were made.

\section{References}

Abt, T. P. (2017). Towards a framework for preventing community violence among youth. Psychology, health \& medicine, 22(sup 1), 266-285.

Bernasco, W., \& Block, R. (2011). Robberies in Chicago: A block-level analysis of the influence of crime generators, crime attractors, and offender anchor points. Journal of Research in Crime and Delinquency, $48(1), 33-57$.

Block, R., \& Davis, S. (1996). The environs of a rapid Transit Station: A focus for street crime or just another risky place. In R. Clarke (Ed.), Preventing mass transit crime: Crime Prevention Studies vol. 6 (pp. 237257). Monsey, NY: Willow Tree.

Bowers, K. J., Johnson, S. D., \& Pease, K. (2004). Prospective hot-spotting: The future of crime mapping? British Journal of Criminology, 44(5), 641-658.

Braga, A. A., Papachristos, A. V., \& Hureau, D. M. (2014). The effects of hot spots policing on crime: An updated systematic review and meta-analysis. Justice Quarterly, 31(4), 633-663.

Brantingham, P., \& Brantingham, P. (1993) Environment, routine and situation: Toward a pattern theory of crime. In Clarke, R., and Felson, M.,. (Eds) Routine activity and rational choice: Advances in criminological theory, vol 5 (pp. 259-294). Piscataway, NJ: Transaction.

Brantingham, P., \& Brantingham, P. (1995). Criminality of place: Crime generators and crime attractors. European Journal on Criminal Policy and Research, 3(3), 5-26.

Brunton-Smith, I., \& Sturgis, P. (2011). Do neighborhoods generate fear of crime? An empirical test using the British crime survey. Criminology, 49(2), 331-369.

Caplan, J. M., Kennedy, L. W., \& Miller, J. (2011). Risk terrain modeling: Brokering criminological theory and GIS methods for crime forecasting. Justice Quarterly, 28(2), 360-381.

Caplan, J. M., Marotta, P., Piza, E. L., \& Kennedy, L. W. (2014a). Risk terrain modeling for strategic and tactical action. Crime Mapping and Analysis News, 1(4), 10-13. 
Caplan, M., Marotta, J., Piza, P. L. E., \& Kennedy, W. L. (2014b). Spatial risk factors of felonious battery to police officers. Policing: An International Journal of Police Strategies \& Management, 37(4), 823-838.

Cohen, L., \& Felson, M. (1979). Social change and crime rate trends: A routine activity approach. American Sociological Review, 44, 588-608.

Ceccato, V., \& Uittenbogaard, A. C. (2014). Space-time dynamics of crime in transport nodes. Annals of the Association of American Geographers, 104(1), 131-150.

Ceccato, V., Uittenbogaard, A., \& Bamzar, R. (2013). Security in Stockholm's underground stations: The importance of environmental attributes and context. Security Journal, 26(1), 33-59.

Cerdá, M., Morenoff, J. D., Hansen, B. B., Tessari Hicks, K. J., Duque, L. F., Restrepo, A., \& Diez-Roux, A. V. (2012). Reducing violence by transforming neighborhoods: A natural experiment in Medellín, Colombia. American Journal of Epidemiology, 175(10), 1045-1053.

Drawve, G. (2016). A metric comparison of predictive hot spot techniques and RTM. Justice Quarterly, 33(3), 369-397.

Drawve, G., Moak, S. C., \& Berthelot, E. R. (2016). Predictability of gun crimes: A comparison of hot spot and risk terrain modelling techniques. Policing and Society, 26(3), 312-331.

Dugato, M. (2013). Assessing the validity of risk terrain modeling in a European city: Preventing robberies in the city of Milan. Crime Mapping, 5(1), 63-89.

Felson, M., Belanger, M., Bichler, G., Bruzinski, C., Campbell, G., Fried, C., Grofik, K., Mazur, I., O’Regan, A., Sweeney, P., Ullman, A., \& Willaims, L. (1996). Redesigning hell: Preventing crime and disorder at the port authority bus terminal. In R. Clarke (Ed.), Preventing mass transit crime: Crime prevention studies, vol. 6 (pp. 5-93). Monsey, NY: Willow Tree.

Gerell, M. (2013). Bränder, skadegörelse, grannskap och socialt kapital. Malmö University Publications In Urban Studies, MAPIUS 11.

Gerell, M., \& Kronkvist, K. (2017). Violent Crime. Collective Efficacy and City-centre effects in Malmö. British Journal of Criminology, 57(5), 1185-1207.

Hart, T. C., \& Miethe, T. D. (2014). Street robbery and public bus stops: A case study of activity nodes and situational risk. Security Journal, 27(2), 180-193.

Hoppe, L., \& Gerell, M. (2018). Near-repeat burglary patterns in Malmö: Stability and change over time. European Journal of Criminology, https://doi.org/10.1177/1477370817751382.

Ivert, AK., Chrysoulakis, A. Kronkvist, K. \& Torstensson-Levander, M. (2013) Malmö områdesundersökning 2012. Lokala problem, brott och trygghet. Rapport från institutionen för kriminologi, Malmö högskola.

Johnson, S. D., Bowers, K. J., \& Pease, K. (2013). Predicting the future or summarising the past? Crime mapping as anticipation. In Smith, M. J., \& Tilley, N. (eds) Crime science: new approaches to preventing and detecting crime (p. 145).Cullompton: Willan.

Johnson, S.D., Bowers, K.J., Birks, D.J. \& Pease, K. (2009). Predictive mapping of crime by ProMap: Accuracy, units of analysis, and the environmental backcloth. In Weisburd, D., Bernasco, W. \& Bruinsma, G. (eds), Putting crime in its place. Units of Analysis in Geographic Criminology. New York Springer.

Johnson, S. D., Davies, T., Murray, A., Ditta, P., Belur, J., \& Bowers, K. (2017). Evaluation of operation swordfish: A near-repeat target-hardening strategy. Journal of Experimental Criminology, 13(4), 505-525.

Kennedy, L. W., Caplan, J. M., \& Piza, E. (2011). Risk clusters, hotspots, and spatial intelligence: Risk terrain modeling as an algorithm for police resource allocation strategies. Journal of Quantitative Criminology, 27(3), 339-362.

Kennedy, L. W., Caplan, J. M., Piza, E. L., \& Buccine-Schraeder, H. (2016). Vulnerability and exposure to crime: Applying risk terrain modeling to the study of assault in Chicago. Applied Spatial Analysis and Policy, 9(4), $529-548$

Loukaitou-Sideris, A. (1999). Hot spots of bus stop crime: The importance of environmental attributes. Journal of the American Planning Association, 65(4), 395-411.

Moreto, W. D., Piza, E. L., \& Caplan, J. M. (2013). “A plague on both your houses?”: Risks, repeats and reconsiderations of urban residential burglary. Justice Quarterly, 31(6), 1102-1126.

Newton, Andrew D. (2008) A study of bus route crime risk in urban areas: the changing environs of a bus journey. Built Environment, 34(1), 88103.

Newton, A (2014). Crime on public transport. Encyclopedia of criminology and criminal justice (pp. 709-720). London: Springer.

Newton, A. and Bowers. K., (2007) The geography of bus shelter damage: The influence of crime, neighbourhood characteristics and land-use. Internet Journal of Criminology.

Piza, E., Feng, S., Kennedy, L., \& Caplan, J. (2016). Place-based correlates of motor vehicle theft and recovery: Measuring spatial influence across neighbourhood context. Urban Studies, https://oi. org/10.1177/0042098016664299.

Ratcliffe, J. (2006). A temporal constraint theory to explain opportunity-based spatial offending patterns. Journal of Research in Crime and Delinquency, 43(3), 261-291. 
Sampson, R. J., Raudenbush, S. W., \& Earls, F. (1997). Neighborhoods and violent crime: A multilevel study of collective efficacy. Science, 277(5328), 918-924.

Sampson, R. J., \& Groves, W. B. (1989). Community structure and crime: Testing social-disorganization theory. American Journal of Sociology, 94(4), 774-802.

Shaw, C. R., \& McKay, H. D. (1942). Juvenile delinquency and urban areas. Chicago: University of Chicago Press.

Smith, M. J., \& Cornish, D. B. (Eds.). (2006). Secure and tranquil travel: Preventing crime and disorder on public transport. Abingdon: Routledge.

St Jean, P. K. (2008). Pockets of crime: Broken windows, collective efficacy, and the criminal point of view. Chicago: University of Chicago Press.

Trickett, A., Osborn, D. R., Seymour, J., \& Pease, K. (1992). What is different about high crime areas? The British Journal of Criminology, 32(1), 81-89.

Weisburd, D., Groff, E. R., \& Yang, S. M. (2014). Understanding and controlling hot spots of crime: The importance of formal and informal social controls. Prevention Science, 15(1), 31-43. 